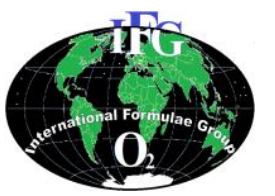

Available online at http://www.ifgdg.org

Int. J. Biol. Chem. Sci. 13(3): 1837-1845, June 2019

International Journal

of Biological and

ISSN 1997-342X (Online), ISSN 1991-8631 (Print)

Original Paper http://ajol.info/index.php/ijbcs http://indexmedicus.afro.who.int

\title{
Etude de stabilité chimique de suspensions buvables à base d'amoxicilline
}

\author{
Jean Simon N'Ghorand Thodhekes YAO ${ }^{1,2^{*}}$, Sawa André Philippe KPAIBE ${ }^{2}$, \\ Nicaise François BONY ${ }^{2}$, Yves Niabith $\mathrm{SOKO}^{1}$ et Michèle $\mathrm{AKE}^{1,2}$ \\ ${ }^{1}$ Institut National de Santé Publique (INSP), BP V 47 Abidjan, Côte d'Ivoire. \\ ${ }^{2}$ UFR Sciences Pharmaceutiques et Biologiques, Université Felix Houphouët Boigny, BP V 34 \\ Abidjan, Côte d'Ivoire. \\ *Auteur correspondant, E-mail: js_thodhekes@yahoo.fr; Tel: (+225) 07239272
}

\section{RESUME}

Les conditionnements multidoses tels les formes reconstituées posent, après ouverture un problème de conservation et de délai d'utilisation. La stabilité chimique portant sur la teneur en principe actif de la forme orale reconstituée d'amoxicilline dosée à $250 \mathrm{mg} / 5 \mathrm{ml}$ a été étudiée sur une durée minimale de traitement de 6 jours consécutifs. Cinq spécialités ont servi à l'étude. Les échantillons ont été reconstitués avec deux qualités différentes d'eaux (eau minérale naturelle et eau de robinet bouillie et refroidie) et conservés à deux températures différentes $\left(5 \pm 3{ }^{\circ} \mathrm{C}\right.$ et $\left.30 \pm 2{ }^{\circ} \mathrm{C}\right)$. La stabilité a été étudiée par chromatographie liquide suivie d'une détection UV à $230 \mathrm{~nm}$. La teneur en amoxicilline dans tous les échantillons était conforme à la norme $(225 \mathrm{mg}$ à $300 \mathrm{mg} / 5 \mathrm{ml})$. La nature de l'eau utilisée n'a pas eu d'influence sur la stabilité. Les échantillons conservés à $30 \pm 2{ }^{\circ} \mathrm{C}$ ont présenté un taux de dégradation en principe actif plus élevé (>6\%). Pour deux marques commerciales, une non-conformité portant sur le volume de reconstitution a été notée. La stabilité chimique des médicaments est donc maintenue sur les 6 jours d'utilisation. Ce type d'étude devrait être étendu aux médicaments sous forme de poudres à reconstituer vendus en Côte d'Ivoire.

(C) 2019 International Formulae Group. All rights reserved

Mots clés: Formes reconstituées, Délai d'utilisation, Chromatographie liquide.

\section{Study of chemical stability of oral suspensions based on amoxicillin.}

\section{ABSTRACT}

Multi-dose packaging such as reconstituted forms pose, after opening a conservation problem and time of use. The chemical stability of the active ingredient content of the reconstituted oral form of amoxicillin at $250 \mathrm{mg} / 5 \mathrm{ml}$ was studied over a minimum treatment period of 6 consecutive days. Five specialties were used for the study. The samples were reconstituted with two different qualities of water (natural mineral water and tap water boiled and cooled) and stored at two different temperatures $\left(5 \pm 3{ }^{\circ} \mathrm{C}\right.$ and $\left.30 \pm 2{ }^{\circ} \mathrm{C}\right)$. Stability was studied by liquid chromatography followed by UV detection at $230 \mathrm{~nm}$. The amoxicillin content in all samples was consistent with the 
standard ( $225 \mathrm{mg}$ to $300 \mathrm{mg} / 5 \mathrm{ml}$ ). The nature of the water used did not affect the stability. Samples stored at $30 \pm 2{ }^{\circ} \mathrm{C}$ showed a higher active ingredient degradation rate (>6\%). For two commercial brands, a nonconformity regarding the reconstitution volume was noted. The chemical stability of the drugs is maintained for 6 days of use. This type of study should be extended to drugs presented as powders to be reconstituted sold in Côte d'Ivoire.

(c) 2019 International Formulae Group. All rights reserved

Keywords: Reconstituted forms, Time of use, Liquid chromatography.

\section{INTRODUCTION}

Les antibiotiques à noyau bêta-lactame appartenant à la famille des pénicillines jouent depuis des décennies un rôle important en médecine humaine en raison de leur large spectre d'activité antimicrobienne, de leur bonne absorption et de leur grande pénétration dans les tissus (Kumar et al., 2014 ; Cha et al., 2004). Ils sont dès lors indiqués dans le traitement de nombreuses infections bactériennes sous diverses manifestations notamment bronchorespiratoires, ORL (otorhino-laryngologie), urogénitales, hépatobiliaires, rénales, gynécologiques, et également lors des septicémies, des méningites ou des endocardites (Vidal, 2010 ; Pilon, 2016). Dans l'arsenal thérapeutique de cette classe, l'amoxicilline est l'un des antibiotiques fréquemment utilisés en pédiatrie, dont les présentations sous forme de poudres reconstituées multidoses sont les plus adaptées aux enfants (Vidal, 2010 ; Jerzsele et Nagy, 2008).

Cependant, ce type de conditionnement peut poser après ouverture, un problème de conservation lié aux conditions favorables de dégradation rapide du principe actif (chaleur, humidité variable, intensité lumineuse) et au délai d'utilisation (Sabot et al., 2001). Dans cette classe des bêta-lactamines, l'amoxicilline, fréquemment prescrite chez les enfants, se dégrade facilement entrainant des risques de sous dosage, de formation de produits de dégradation etc. (Lahlou et al., 2009).

De nombreuses suspensions d'antibiotiques reconstituées doivent être conservées au réfrigérateur afin d'obtenir des avantages optimaux (Alzomor et al., 2016). Cependant, ces conditions de conservation ne sont souvent pas clairement spécifiées.
Il nous est donc paru opportun, dans la présente étude, d'évaluer la stabilité chimique de l'amoxicilline dans certaines formes reconstituées avec deux qualités d'eau (eau minérale, eau de robinet) puis conservées à deux températures (ambiante et réfrigéré). Un contrôle préalable du volume nécessaire à la reconstitution a été réalisé. Cette étude s'est déroulée sur une période de six (6) jours correspondant à la durée moyenne d'un traitement.

\section{MATERIEL ET METHODES}

\section{Appareillage}

Le dosage de l'amoxicilline s'est effectué sur un chromatographe liquide (Shimadzu, France) équipé d'une pompe (DGU-20A5), d'un système d'injection automatique (LC-20AT), d'un détecteur UVVisible réglé à $230 \mathrm{~nm}$ (SPD $20 \mathrm{AT}$ ) et d'un enregistreur intégrateur (CTO-20A).

L'étude a nécessité également un agitateur magnétique (Vortex Génie II, USA) ; un $\mathrm{pH}$ mètre (Thermo-Orion, USA); une étuve (Jouan, USA) ; une balance de précision (Sartorius, Allemagne) et un dispositif de filtration sur Buchner pour le dégazage.

\section{Réactifs et solutions de travail}

- Les réactifs utilisés étaient de qualité analytique :

$\begin{array}{rrr}\text { Acétonitrile pour } & \text { chromatographie } \\ \text { liquide } & \text { (Scharlau, } & \text { Espagne); }\end{array}$
Dihydrogénophosphate de potassium (Merck, Allemagne); Hydroxyde de potassium (Merck, Allemagne) et Eau. Le produit de référence était l'amoxicilline trihydratée (Sigma-Aldrich, Allemagne) de titre : 100,2\% $(858,7 \mu \mathrm{g} / \mathrm{mg})$.

\footnotetext{
- Les solutions de travail préparées étaient:
} 
- Solution tampon phosphate 0,02 M, pH 5 ;

- Solution d'hydroxyde de potassium $(1 \%)$;

- Solution de référence d'amoxicilline à $0,2 \mathrm{mg} / \mathrm{ml}$;

\section{Echantillonnage}

L'échantillonnage a été constitué de cinq (5) marques commerciales de poudres pour suspensions buvables à base d'amoxicilline sélectionnées après une préenquête réalisée sur une période de 30 jours dans deux officines de pharmacie de la place. Les cinq marques commerciales les plus vendues ont été retenues.

Ces marques codifiées par les lettres A, $\mathrm{B}, \mathrm{C}, \mathrm{D}$ et $\mathrm{E}$ étaient dosées chacune à $250 \mathrm{mg}$ d'amoxicilline pour $5 \mathrm{ml}$. Quatre (4) boites de chacune des cinq marques retenues ont été nécessaires pour l'analyse, soit un total de 20 échantillons.

\section{Méthodologie}

Chacun des échantillons a été reconstitué avec 2 différentes qualités d'eaux puis conservé à 2 températures différentes $\left(5 \pm 3{ }^{\circ} \mathrm{C}\right.$ et $\left.30 \pm 2{ }^{\circ} \mathrm{C}\right)$. La stabilité a été étudiée en déterminant la teneur en amoxicilline pendant 6 jours consécutifs par chromatographie liquide en phase inverse après validation de la méthode d'analyse.

Le volume d'eau nécessaire pour la reconstitution a été évalué pour chaque marque commerciale.

Les pourcentages de dégradation ont été calculés après les 6 jours de reconstitution selon la formule suivante :

Pourcentage de dégradation = $\frac{\text { Teneur à } J 6(\mathrm{mg} / 5 \mathrm{ml})-\text { Teneur à } J 1(\mathrm{mg} / 5 \mathrm{ml})}{\text { Teneur à } J 6(\mathrm{mg} / 5 \mathrm{ml})} \times$

\section{$100 \quad(1)$}

\section{Mode de reconstitution des suspensions}

Deux qualités d'eaux ont été utilisées :

- Eau minérale naturelle (EMN) ;

- Eau de robinet bouillie et refroidie (ER).

Le volume d'eau utilisé était de $60 \mathrm{ml}$ comme indiqué sur le conditionnement, correspondant ainsi à 12 doses de $5 \mathrm{ml}$ de suspension selon le fabricant. Après reconstitution, les flacons ont été vigoureusement agités.

\section{Températures de conservation}

Chaque suspension préparée à l'aide de l'une ou l'autre qualité d'eaux a été conservée à deux températures différentes:

- $5 \pm 3{ }^{\circ} \mathrm{C}$

- $\quad 30 \pm 2{ }^{\circ} \mathrm{C}$

Ces conditions représentent les conditions de stockage à domicile (Nwokoye, 2012).

\section{Préparation de l'extrait à analyser}

Un millilitre de la suspension reconstituée a été prélevé dans une fiole jaugée de $100 \mathrm{ml}$. La prise d'essai a été diluée dans $15 \mathrm{ml}$ de tampon phosphate $\mathrm{pH} 5,0 . \mathrm{La}$ solution obtenue a été soumise à agitation mécanique vigoureuse pendant $10 \mathrm{~min}$, puis complétée jusqu'au trait de jauge avec le tampon pH 5,0. La solution a été homogénéisée par agitation durant cinq minutes et filtrée. La solution obtenue a constitué l'extrait à analyser par chromatographie liquide (USP 31, 2008).

\section{Conditions d'analyse chromatographique}

Les conditions d'analyse retenues sont tirées des travaux de Perez - Lozan et al. (2006):

- Phase mobile: tampon phosphate 0,02 M pH 5 / acétonitrile (95/5, v/v) ;

- Phase stationnaire C18, $250 \times 4,6 \mathrm{~mm}$ x $5 \mu \mathrm{m}$;

- Débit à $0,8 \mathrm{ml} / \mathrm{min}$;

- Détection UV à 230 nm.

\section{Analyses statistiques}

Le traitement des données a été réalisé avec Excel 2013.

Les teneurs moyennes en amoxicilline ont été comparées en utilisant le test de Student.

Le test $\mathrm{T}$ de Student a été utilisé au seuil de signification de $5 \%$ pour la comparaison des échantillons aux différentes températures de conservation avec les différentes qualités d'eaux de reconstitution.

\section{RESULTATS}

Trois des cinq marques commerciales sélectionnées ont nécessité $60 \mathrm{ml}$ d'eau pour leur reconstitution contre $42 \mathrm{ml}$ d'eau pour les 
deux autres. Les teneurs en amoxicilline des échantillons d'une même marque varient peu au cours du temps selon la qualité d'eau utilisée.

La teneur en amoxicilline des échantillons conservés à température ambiante et à température réfrigérée est présentée dans le Tableau 1.

Durant cette période minimale de traitement de 6 jours, il est à noter une diminution progressive de la teneur en amoxicilline qui reste dans l'intervalle de conformité, se situant entre 225 et $300 \mathrm{mg}$. Ce taux reste donc conforme aux normes (90 à 120\%) (USP 31, 2008).

L'évolution au cours du temps de la quantité en amoxicilline dans les 5 marques commerciales après reconstitution pendant 6 jours est illustrée par les Figures 1 à 4.

La teneur en amoxicilline des différents échantillons reconstitués diminuent au cours du temps quelles que soient les conditions de traitement.

L'analyse révèle une diminution marquée de la teneur en amoxicilline dans les échantillons conservés à température ambiante comparativement à ceux conservés à $5 \pm 3{ }^{\circ} \mathrm{C}$ (Figure 1, 2, 3, 4).
Après 6 jours de reconstitution, le taux de dégradation en amoxicilline était inférieur à $18 \%$ dans tous les échantillons. Ces résultats sont présentés dans le Tableau 1.

La teneur maximale en amoxicilline retrouvée est de $299,90 \mathrm{mg}$. Au sixième jour, les teneurs les plus faibles $(226,35 \mathrm{mg}$; $227,95 \mathrm{mg} ; 228,15 \mathrm{mg}$ ) sont retrouvées dans le cas des échantillons conservés à température ambiante. Les différentes suspensions orales ne contiennent pas moins de $90 \%$ et pas plus de $120 \%$ de quantité en amoxicilline.

Le Tableau 2 révèle le niveau de significativité statistique en croissant les paramètres de conservation et de reconstitution.

Il n'y a pas de différence significative au seuil $\mathrm{p}<5 \%$, concernant la qualité de l'eau entre les échantillons reconstitués avec l'eau minérale et ceux reconstitués avec l'eau de robinet. Il y a par contre une différence significative concernant le mode de conservation entre les échantillons conservés à température ambiante et ceux conservés au réfrigérateur au seuil $\mathrm{p}<5 \%(\mathrm{p}=0,227$ à $5 \pm 3$ ${ }^{\circ} \mathrm{C}$ et $\mathrm{p}=0,443$ à $30 \pm 2 \quad{ }^{\circ} \mathrm{C}$ ) (Tableau 2).

Tableau 1 : Evaluation des teneurs des échantillons d'amoxicilline suspension.

\begin{tabular}{|c|c|c|c|c|c|}
\hline \multirow{2}{*}{$\begin{array}{l}\text { Température } \\
\text { de } \\
\text { conservation }\end{array}$} & \multirow{2}{*}{$\begin{array}{c}\text { Eau de } \\
\text { reconstitutio } \\
\mathbf{n}\end{array}$} & \multirow[t]{2}{*}{ Spécialité } & \multicolumn{2}{|c|}{ Concentration } & \multirow{2}{*}{$\begin{array}{l}\text { Pourcentage de } \\
\text { dégradation }(\%)\end{array}$} \\
\hline & & & $\mathrm{J}_{1}(\mathrm{mg} / \mathbf{5} \mathrm{ml})$ & $\mathrm{J}_{6}(\mathrm{mg} / \mathbf{5} \mathrm{ml})$ & \\
\hline \multirow{10}{*}{$\begin{array}{c}\left(5 \pm 3{ }^{\circ} \mathrm{C}\right) \\
\text { (Réfrigéré) }\end{array}$} & \multirow{5}{*}{$\begin{array}{c}\text { Eau minérale } \\
\text { naturelle } \\
(\mathrm{EMN}\end{array}$} & A & $262,30 \pm 0,41$ & $249,95 \pm 0,19$ & 4,70 \\
\hline & & B & $299,90 \pm 0,63$ & $290,50 \pm 0,51$ & 3,13 \\
\hline & & $\mathrm{C}$ & $267,60 \pm 0,28$ & $256,55 \pm 0,45$ & 4,12 \\
\hline & & $\mathrm{D}$ & $262,85 \pm 0,46$ & $254,75 \pm 0,02$ & 3,07 \\
\hline & & $\mathrm{E}$ & $260,30 \pm 0,38$ & $247,15 \pm 0,83$ & 5,05 \\
\hline & \multirow{5}{*}{$\begin{array}{l}\text { Eau de robinet } \\
\text { (ER) }\end{array}$} & A & $259,85 \pm 0,26$ & $249,10 \pm 0,08$ & 4,14 \\
\hline & & B & $293,40 \pm 0,50$ & $285,05 \pm 0,30$ & 2,85 \\
\hline & & $\mathrm{C}$ & $259,00 \pm 0,52$ & $249,55 \pm 0,47$ & 3,65 \\
\hline & & $\mathrm{D}$ & $259,05 \pm 0,05$ & $249,30 \pm 0,01$ & 3,76 \\
\hline & & $\mathrm{E}$ & $263,85 \pm 0,03$ & $254,60 \pm 0,67$ & 3,50 \\
\hline \multirow{4}{*}{$\begin{array}{c}\text { Température } \\
\text { ambiante }(30 \pm 2 \\
\left.{ }^{\circ} \mathrm{C}\right)\end{array}$} & \multirow{4}{*}{$\begin{array}{c}\text { Eau minérale } \\
\text { naturelle } \\
(\mathrm{EMN}\end{array}$} & A & $262,80 \pm 0,37$ & $231,20 \pm 0,19$ & 12,02 \\
\hline & & B & $298,50 \pm 0,09$ & $270,70 \pm 0,28$ & 9,32 \\
\hline & & $\mathrm{C}$ & $255,00 \pm 0,22$ & $227,95 \pm 0,41$ & 10,60 \\
\hline & & $\mathrm{D}$ & $272,75 \pm 0,25$ & $226,35 \pm 0,17$ & 17,01 \\
\hline
\end{tabular}




\begin{tabular}{ccccc} 
& E & $260,15 \pm 0,39$ & $244,20 \pm 0,38$ & 6,13 \\
Eau de robinet & A & $260,05 \pm 0,09$ & $228,15 \pm 0,11$ & 12,27 \\
(ER) & B & $297,90 \pm 0,57$ & $274,65 \pm 0,26$ & 7,81 \\
& C & $251,30 \pm 0,19$ & $233,45 \pm 0,37$ & 7,10 \\
& D & $255,35 \pm 0,34$ & $232,75 \pm 0,06$ & 8,85 \\
& E & $257,75 \pm 0,03$ & $242,15 \pm 0,49$ & 6,07 \\
\hline
\end{tabular}

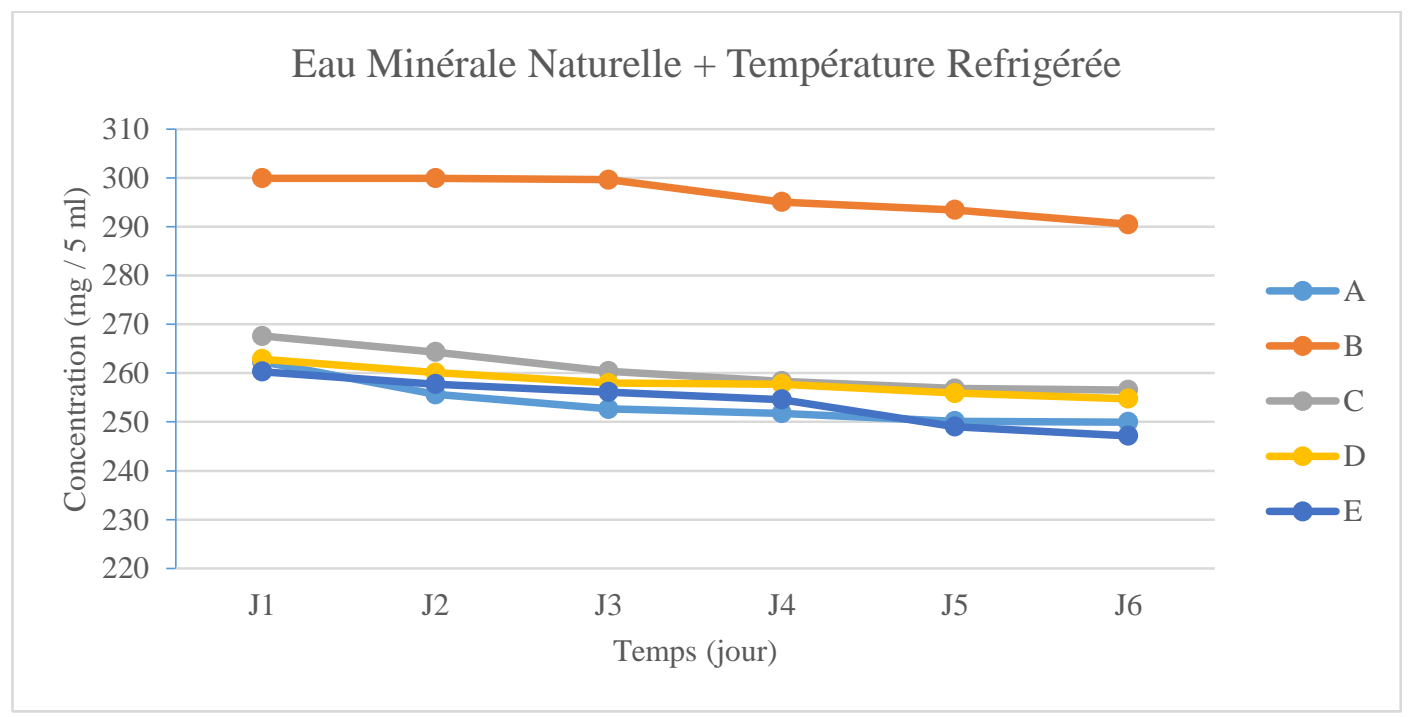

Figure 1: Courbes d'évolution des teneurs en amoxicilline des différents échantillons reconstitués avec de l'eau minérale naturelle et conservés à $5 \pm 3{ }^{\circ} \mathrm{C}$.

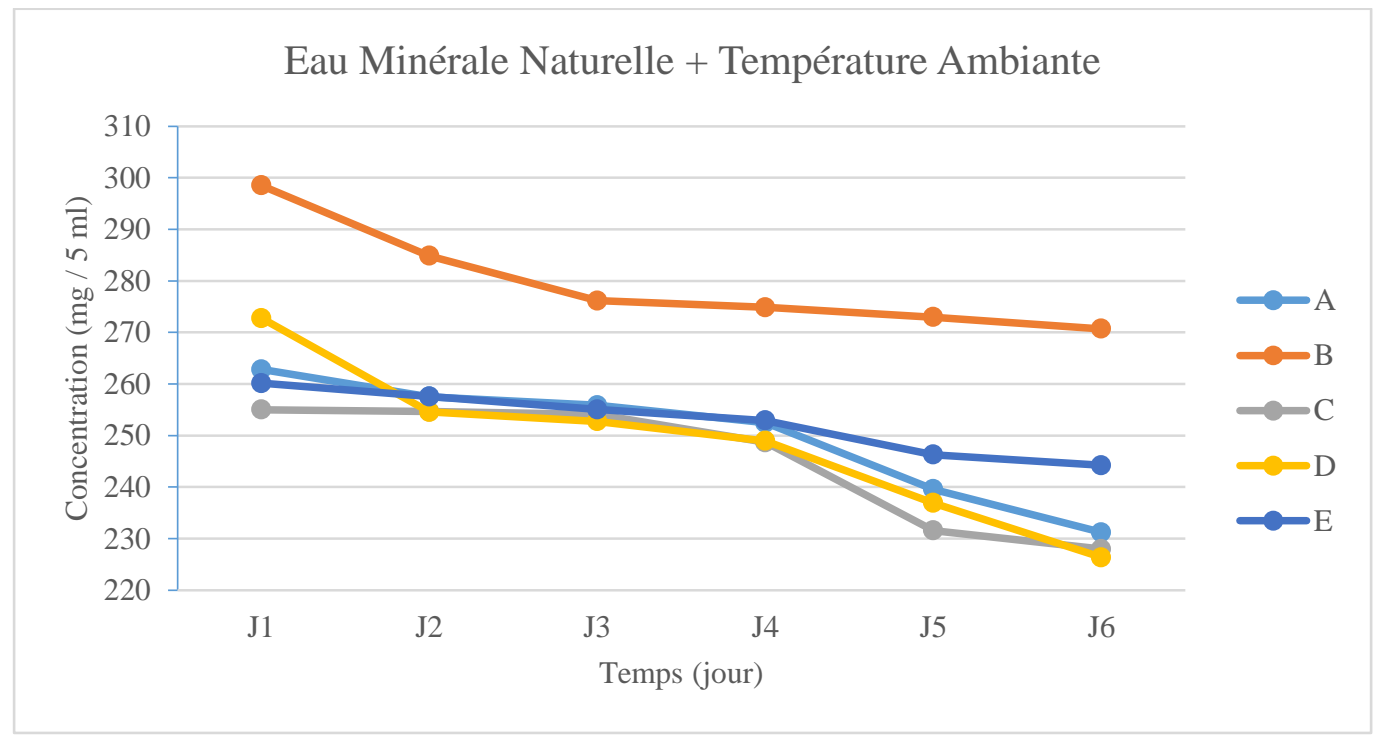

Figure 2: Courbes d'évolution des teneurs en amoxicilline des différents échantillons reconstitués avec de l'eau minérale naturelle et conservés à $30 \pm 2{ }^{\circ} \mathrm{C}$. 


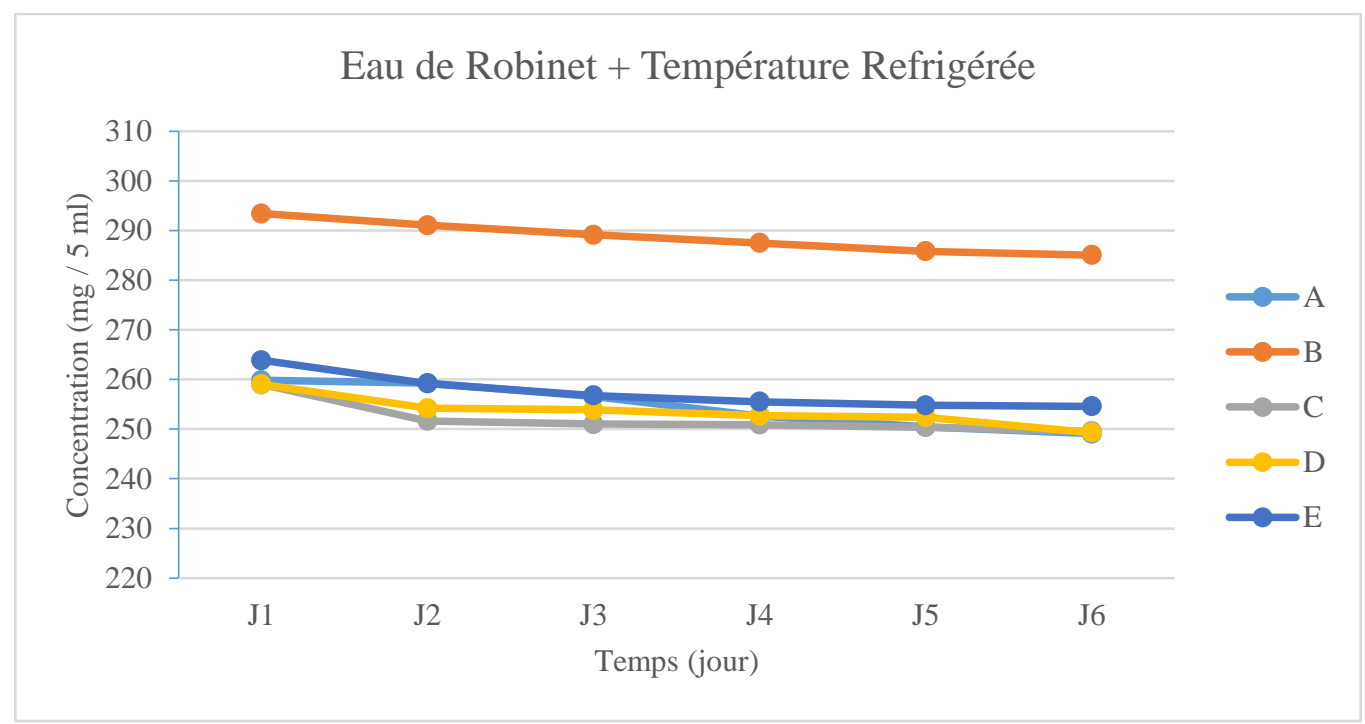

Figure 3 : Courbes d'évolution des teneurs en amoxicilline des différents échantillons reconstitués avec de l'eau de robinet et conservés à $5 \pm 3{ }^{\circ} \mathrm{C}$.

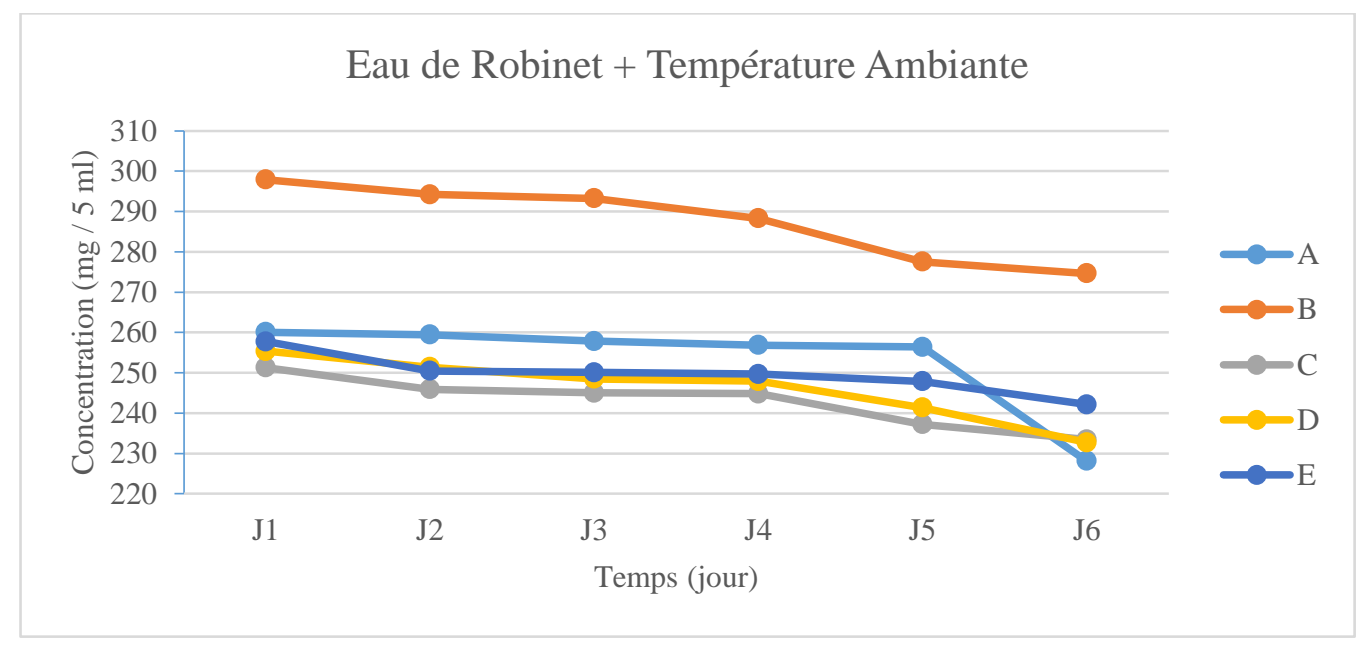

Figure 4: Courbes d'évolution des teneurs en amoxicilline des différents échantillons reconstitués avec de l'eau de robinet et conservés à $30 \pm 2{ }^{\circ} \mathrm{C}$.

Tableau 2: Analyse statistique ; test échantillons appariés.

\begin{tabular}{lcc}
\hline $\begin{array}{l}\text { Paires } \\
\text { (Température de conservation + Eau de reconstitution) }\end{array}$ & $\begin{array}{c}\text { Test T } \\
(\mathbf{p})\end{array}$ & Interprétation \\
\hline$\left(5 \pm 3{ }^{\circ} \mathrm{C}+\mathrm{EMN}{ }^{\square}\right)-\left(5 \pm 3^{\circ} \mathrm{C}+\mathrm{ER}\right.$ & 0,227 & Non significatif \\
\hline$\left(30 \pm 2{ }^{\circ} \mathrm{C}+\mathrm{EMN}\right)-\left(30 \pm 2{ }^{\circ} \mathrm{C}+\mathrm{ER}\right)$ & 0,443 & Non significatif \\
\hline$\left(5 \pm 3{ }^{\circ} \mathrm{C}+\mathrm{EMN}\right)-\left(30 \pm 2{ }^{\circ} \mathrm{C}+\mathrm{EMN}\right)$ & 0,044 & Significatif \\
\hline$\left(5 \pm 3{ }^{\circ} \mathrm{C}+\mathrm{ER}\right)-\left(30 \pm 2{ }^{\circ} \mathrm{C}+\mathrm{ER}\right)$ & 0,014 & Significatif \\
\hline$:$ Eau minérale naturelle & & \\
$:$ Eau de robinet & &
\end{tabular}




\section{DISCUSSION}

\section{Volume de reconstitution}

La reconstitution des poudres pour suspension buvable d'amoxicilline nécessite un volume d'eau de $60 \mathrm{ml}$, confirmée par le trait de jauge indiqué sur le flacon.

Pour les échantillons A, D et E, les $60 \mathrm{ml}$ d'eau utilisés pour la reconstitution correspondent effectivement au trait de jauge. Par contre, pour reconstituer les échantillons $B$ et $\mathrm{C}$, seulement $42 \mathrm{ml}$ d'eau ont été nécessaires pour atteindre le niveau de jauge. Ces $42 \mathrm{ml}$ d'eau correspondent à 8 doses de principe actif.

Dans ces deux derniers cas, il manque un volume de $18 \mathrm{ml}$ de suspension pour assurer la totalité du traitement sur les six jours de traitement préconisés. Cette observation pourrait impliquer la nécessité d'achat de flacon(s) supplémentaire(s) pour couvrir la durée de la prescription entrainant ainsi une augmentation du coût de la prise en charge. Ces résultats sont en inadéquation avec les volumes indiqués dans les notices de forme orale reconstituée d'amoxicilline dosée à $250 \mathrm{mg} / 5 \mathrm{ml}$ (Vidal, 2010). Markman et al. (2014) dans ses travaux sur la qualité et la stabilité de suspension reconstituée d'amoxicilline dosé à $250 \mathrm{mg} / 5 \mathrm{ml}$ n'a relevé aucune anomalie du volume de reconstitution sur les sept spécialités analysées.

Le trait de jauge n'indiquant pas le niveau correct de remplissage s'expliquerait par un défaut dans le processus de fabrication des flacons ou une fraude sur la quantité réelle de matière première. Cette fraude consisterait à réduire à la fois la quantité d'eau utilisée et la quantité de matière première afin d'obtenir après analyse de la forme à reconstituer, des concentrations en principe actif conformes à celle déclarée.

Selon Pinel (2014), les contrefaçons se retrouvent aussi bien dans le circuit formel qu'informel.

\section{Stabilité du principe actif}

La stabilité est définie comme la capacité d'une substance médicamenteuse ou médicament à rester dans les limites des spécifications établies pour maintenir son identité, sa qualité et pureté.

Elle détermine la teneur en substance médicamenteuse ou produit de dégradation due à l'exposition à une variété de conditions telles que la température, l'humidité, la lumière et le matériau d'emballage sur une période définie (Alzomor et al., 2016).

La dégradation des suspensions peut résulter soit de la température ou de la qualité de l'eau utilisée pour la reconstitution, soit de conditions de stockage inappropriées (Nwokoye et al., 2012; Stanley et Igala, 2017).

\section{Eaux utilisées pour la reconstitution}

Il n'y a pas de différence significative entre les échantillons reconstitués avec l'eau minérale et ceux reconstitués avec l'eau de robinet au seuil de signification $\mathrm{p}<5 \%$ (test $\mathrm{T}$ Student).

L'eau de robinet et l'eau minérale naturelle peuvent donc être conseillées pour la reconstitution des poudres pour suspension buvable à base d'amoxicilline.

\section{Température de conservation}

La teneur en amoxicilline des suspensions reconstituées conservées à température ambiante décroit plus vite que celle des échantillons conservés entre $5 \pm 3{ }^{\circ} \mathrm{C}$ (Figure 1,2) et (Figure 3, 4).

Le taux de dégradation d'amoxicilline en 6 jours pour les échantillons conservés au réfrigérateur reste inférieur ou égal à 5\%, donc conforme aux normes spécifiées (norme $\leq 10 \%$ ) (USP 31, 2008).

Les échantillons conservés à température ambiante ont au moins un taux de dégradation supérieur à $6 \%$ allant jusqu'à plus de $17 \%$ (Tableau 1). Alforjani et Kamour (2019) trouvent après 4 jours de reconstitution, un taux de dégradation en amoxicilline inférieure à $17 \%$.

Ces variations de la teneur en amoxicilline observées restent dans l'intervalle exigé. Les échantillons stockés sous ces deux conditions de température restent stables tout au long de cette période.

Des facteurs humains et erreurs systémiques peuvent également influencer la stabilité du principe actif (Abbou, 2016).

Il existe une différence significative de teneur en amoxicilline lorsque l'on compare une même marque reconstituée avec la même qualité d'eau mais conservée à différentes températures $(\mathrm{p}=0,044$ quand l'eau utilisée est l'eau minérale et $\mathrm{p}=0,014$ avec l'eau de robinet) (Tableau 2). Les teneurs en 
amoxicilline des différents échantillons restent malgré tout dans les normes spécifiées de la pharmacopée américaine (USP 31, 2008).

$\mathrm{La}$ conservation à température ambiante est un facteur de dégradation rapide de l'amoxicilline.

\section{Traitement appliqué aux échantillons (eau de reconstitution et température de conservation)}

L'amoxicilline conservé à une température comprise de $5 \pm 3{ }^{\circ} \mathrm{C}$ reconstitués avec l'eau de robinet bouillie et refroidie présente une meilleure stabilité sur les 6 jours d'utilisation (Tableau 1). Ce constat se rapproche des travaux de Alzomor et al. (2016) qui trouvent l'amoxicilline stable durant 7 jours dans les échantillons contenant l'association amoxicilline et acide clavulanique, lorsqu'ils sont conservés à $5 \pm 3$ ${ }^{\circ} \mathrm{C}$.

Naidoo et al. (2006) montrent qu'au $7^{\text {ème }}$ jour, plus de $11 \%$ de l'amoxicilline sont dégradés lorsque conservé à une température comprise entre $20^{\circ} \mathrm{C}$ et $+25^{\circ} \mathrm{C}$. Ce constat est en accord avec les variations de teneurs de certains échantillons de notre étude.

Selon Markman et al. (2014), 83\% des formes orales d'amoxicilline étudiées ont un taux de dégradation inférieur à $10 \%$. Ce constat se rapproche des observations de notre étude où $80 \%$ des échantillons analysés ont un taux de dégradation également inférieur à 10\% (Markman et al., 2014).

Les suspensions conservées à température ambiante sont moins stables.

\section{Conclusion}

L'étude a permis d'évaluer la stabilité en cours d'utilisation des médicaments à base d'amoxicilline présentés sous forme de poudre pour suspension buvable commercialisés en Côte d'Ivoire. Cette étude a porté sur l'analyse des suspensions reconstituées avec deux qualités différentes d'eaux à deux températures de conservation (température ambiante et température réfrigérée) sur 6 jours. Elle a permis d'apprécier la durée d'utilisation dans des conditions de conservation ou d'entreposage. Parmi les cinq marques commerciales analysées, une nonconformité a été notée, portant sur le volume de reconstitution pour deux marques commerciales. La teneur en amoxicilline a été évaluée sur six jours d'utilisation après reconstitution. Elle a montré que, quelle que soit la température de conservation, cette teneur reste maintenue dans les normes. Les formes reconstituées conservées au réfrigérateur ont montré une meilleure stabilité de la teneur que celles conservées à température ambiante quelle que soit l'eau utilisée. Ce type d'étude devrait être étendu à l'ensemble des formes à reconstituer commercialisées en zone tropicale.

\section{CONFLIT D'INTERETS}

Les auteurs déclarent qu'il n'y a aucun conflit d'intérêts pour ce travail.

\section{CONTRIBUTIONS DES AUTEURS}

JSNTY est l'investigateur principal.

SAPK a contribué à l'élaboration du protocole. NFB a donné des orientations sur les travaux et a participé à la révision du protocole utilisé. YNS a été responsable de l'expérimentation et en a assuré son authenticité. MA est responsable du laboratoire où se sont tenus les travaux. Elle a validé le protocole expérimental, supervisé le travail, participé à la rédaction du manuscrit. Elle a été le responsable scientifique de cette étude.

\section{REMERCIEMENTS}

Le Laboratoire de Nutrition de l'Institut National de Santé Publique.

\section{REFERENCES}

Abbou S. 2016. Etude de Stabilité et Contrôle Qualité sur «Augmentin PPSB $60 \mathrm{ml}$ ». Mémoire de Master, Université Abderrahmane Mira de Béjaia, Algérie, p.50

Alforjany EA, Kamour RM. 2019. Effect of temperature of water used for reconstitution on stability of antibiotic dry suspension. World J. Pharm. Pharm. Sci., 8(2): 116-126. DOI : 10.20959/wjpps20192-13123

Alzomor AK, Noman NM and Alfarzai SA. 2016. Stability study for three brands coamoxiclav oral suspension $(312.5 / 5 \mathrm{ml})$ after reconstitution at refrigerator (2-8 
${ }^{\circ}$ C). European J. Biomed. Pharm. Sci., 3(6): 203-209.

Bouchara C, Delandre E, Dory M, Philippe S, Dieu B, Varin R. 2012. Outil d'aide à l'administration: conservation après ouverture des formes multidoses. Le Pharmacien Hospitalier et Clinicien, 47(1): S1-S102. DOI: https://doi.org/10.1016/j.phclin.2011.12. 179

Cha R, Rybak MJ. 2004. Pulsatile delivery of amoxicillin against Streptococcus pneumonia. J. Antimicrob. Chemother., 54 : 1067-1071. DOI: https://doi.org/10.1093/jac/dkh452

Jerzsele A, Nagy G. 2009. The stability of amoxicillin trihydrate and potassium clavulanate combination in aqueous solutions. Acta Vet. Hung., 57 (4): 485493. DOI : 10.1556/AVet.57.2009.4.3

Kumar R, Anvesh MS, Khan MS, Moin A, Gowda DV. 2014. Formulation and Evaluation of Two-Pulse Drug Delivery System of Amoxicillin Trihydrate. Trop J Pharm Res., 13 (10) : 1593. DOI : http://dx.doi.org/10.4314/tjpr.v13i10.4

Lahlou AI, Chegri M, L'Kassmi H. 2009. Epidémiologie et résistance aux antibiotiques des entérobactéries isolées d'infections urinaires à l'hôpital militaire Moulay-Ismail de Meknès. Antibiotiques ed. Elsevier Masson, 11 (2): 90-96. DOI: $10.1016 / j$.antib.2008.10.004

Markman BEO, Koschtschak MRW, Meihuey EW, Rosa PCP. 2014. Evaluation of the quality and stability of amoxicillin oral suspension. J. App. Pharm. Sci., 4 (07): 38-40. DOI: 10.7324/JAPS.2014.40706

Naidoo KK, Nompuku P, Mkalali SN, Shabangu K, Nkabinde L, Singh V. 2006. Post-marketing stability surveillance: Amoxicillin. SA Fam Pract J, 48(6) : 14-14b. DOI: http://dx.doi.org/10.1080/20786204.200 6.10873401

Nwokoye P, Oyetunde O, Akinleye M. 2012. Stability of reconstituted amoxicillin clavulanate potassium under simulated in-home storage conditions. J. App. Pharm. Sci., 02(01): 28-31.

Perez-Lozano P, Garcia-M, Orriols A, Miñarr M, Ticó JR. 2006. Stability evaluation of amoxicillin in a solid premix veterinary formulation by monitoring the degradation products through a new HPLC analytical method. J. Pharm. Biomed. Anal., 42: 192-199. DOI : https://doi.org/10.1016/j.jpba.2006.04.0 24

Pilon S. 2016. Médicaments essentiels - guide pratique d'utilisation. (Ed. 2016) MSF : Paris.

Pinel J. 2014. Les médicaments de contrefaçons et sous-standards : un danger de mort, exceptionnel dans les pays industrialisés, commun dans les pays en développement. http://apps.who.int/medicinedocs/docum ents/s23072fr/s23072fr.pdf

Stanley CN, Igala SE. 2017. Effect of different storage conditions on the stability and efficacy of Some reconstituted oral antibiotic suspensions sold in port harcourt. Nigeria J. Pharm. Res. Int., 20(3): 1-10. DOI : 10.9734/JPRI/2017/38553

United States Pharmacopeial Convention. 2008. The United States pharmacopeia: USP 31; The National formulary: NF 26, Rockville, Maryland.

Vidal. 2010. Dictionnaire Médical. Edition Vidal : France. 